\title{
Inclined Jet in Crossflow Interacting with a Vortex Generator
}

\author{
K. B. M. Q. Zaman* \\ NASA John H. Glenn Research Center at Lewis Field, Cleveland, Ohio 44135 \\ D. L. Rigby $\ddagger$ \\ ASRC Aerospace, Inc., Greenbelt, Maryland 20770 \\ and \\ J. D. Heidmann \\ NASA John H. Glenn Research Center at Lewis Field, Cleveland, Ohio 44135
}

DOI: $10.2514 / 1.49742$

\begin{abstract}
An experiment is conducted on the effectiveness of a vortex generator in preventing liftoff of a jet in crossflow, with possible relevance to film-cooling applications. The jet issues into the boundary layer at an angle of $20^{\circ}$ to the freestream. The effect of a triangular ramp-shaped vortex generator is studied while varying its geometry and location. Detailed flowfield properties are obtained for a case in which the height of the vortex generator and the diameter of the orifice are comparable with the approach boundary-layer thickness. The vortex generator produces a streamwise vortex pair with a vorticity magnitude 3 times larger (and of opposite sense) than that found in the jet in crossflow alone. Such a vortex generator appears to be most effective in keeping the jet attached to the wall. The effect of parametric variation is studied mostly from surveys 10 diameters downstream from the orifice. Results over a range of jet-to-freestream momentum flux ratio $(1<J<11)$ show that the vortex generator has a significant effect even at the highest $J$ covered in the experiment. When the vortex generator height is halved, there is a liftoff of the jet. On the other hand, when the height is doubled, the jet core is dissipated due to larger turbulence intensity. Varying the location of the vortex generator, over a distance of three diameters from the orifice, is found to have little impact. Rounding off the edges of the vortex generator with the increasing radius of curvature progressively diminishes its effect. However, allowing for a small radius of curvature may be quite tolerable in practice.
\end{abstract}

\section{Introduction}

A VORTEX generator (VG) geometry for boundary-layer flow control, sometimes referred to as a microramp, has been addressed in recent studies [1-3]. With certain orientation, the VG produces a pair of streamwise vortices for which the sense is such that there is a downwash between the two vortices and the freestream fluid is ingested and drawn toward the wall. This sense is opposite to that of the bound vortex pair occurring in the jet in crossflow (JICF) that tends to cause the jet to lift away from the wall. Thus, placing the VG downstream of an inclined JICF, typical of a film-cooling application, has the promise of keeping the jet attached to the wall via vorticity cancellation. The basic concept is not new and has been tried before with other methods of streamwise vortex generation (e.g., $[4,5])$. The present VG geometry for film-cooling purposes was explored in the computational fluid dynamics study of [1] that showed promising results. This prompted the current investigation to further explore the potential of the method. The basic shape of the VG, as well as the inclination of the isolated JICF, is chosen to be somewhat different from those used in [1], for reasons explained in the following section. Detailed flowfield data are acquired while varying the location and height of the VG as well as the momentum flux ratio of the JICF. Furthermore, since a sharp-edged VG may not be easy to implement in practice, the effect of rounding off its edges is also examined. Hot-wire anemometry is used to measure mean

Presented as Paper 2010-0088 at the 48th AIAA Aerospace Sciences Meeting, Orlando, FL, 4-7 January 2010; received 5 March 2010; revision received 29 June 2010; accepted for publication 1 July 2010. This material is declared a work of the U.S. Government and is not subject to copyright protection in the United States. Copies of this paper may be made for personal or internal use, on condition that the copier pay the $\$ 10.00$ per-copy fee to the Copyright Clearance Center, Inc., 222 Rosewood Drive, Danvers, MA 01923; include the code 0748-4658/10 and \$10.00 in correspondence with the CCC

*Inlet and Nozzle Branch, Aeropropulsion Division. Associate Fellow AIAA.

Contract Employee, Turbomachinery and Heat Transfer Branch, NASA John H. Glenn Research Center at Lewis Field, Cleveland, OH 44135.

†Turbomachinery and Heat Transfer Branch, Aeropropulsion Division. Member AIAA. velocity, turbulence, and streamwise vorticity while varying different parameters. Key results are discussed in this paper.

\section{Experimental Facility and Procedure}

The measurements are carried out in a low-speed open-loop wind tunnel having a 20 in. high by 30 in. wide test section. The experimental arrangement and the VG models are shown in Fig. 1. The jet orifice is a round hole of diameter $d=0.75 \mathrm{in}$. inclined at an angle of $20^{\circ}$. This geometry of the orifice is chosen because an earlier study was conducted with it [6]. The orifice plate, flush mounted on the floor of the test section, has a thickness of $1 \mathrm{in}$. The supply for the jet is shop air that goes through a flow conditioning screen into a settling chamber before issuing into the test section through the orifice [6]. The jet velocity $U_{j}$ is monitored by a pressure transducer across an orifice meter in the line. The flow rate is calibrated by measuring the velocity (using a pitot probe) at the exit of a 0.75 -in.diam orifice that is cut perpendicular to the surface of another identical orifice plate. As it will be seen in the following, the velocity distribution at the exit of the inclined orifice in highly nonuniform; thus, $U_{j}$ represents an average velocity.

The VG and its location relative to the orifice are shown schematically in Fig. 2. Detailed surveys are conducted for the VG model denoted $\mathrm{H} 0$ (or $\mathrm{R} 0$ ) in Fig. 1c. It has dimensions $L, S$, and $H$ of $1.435,1.18$, and 0.561 in., respectively. The half-angle at the apex $\left(22.5^{\circ}\right)$ is somewhat smaller than that used in [1] but closer to that used in [2]. Various other VG models used in the study are shown in Fig. 1c. Their essential dimensions are listed in Table 1 . Note that, when varying the radius of curvature of the edges of the VG (models R0 through R4), the maximum height and the frontal area blockage were kept constant. The limiting model in this series is R4 that is a hemisphere with a small cylindrical base. Most of the data taken are for the VG placed with its apex upstream (film-cooling configuration), as shown in Figs. 1 and 2. Limited results taken with a reversed (mixing enhancement) ${ }^{-}$configuration, with apex downstream, are also discussed at the end of the paper.

The approach boundary-layer profiles are shown in Fig. 3. The turbulent boundary-layer thickness $(\delta \approx 0.6 d)$ is comparable to the height of the R-series models $(H=0.75 d)$. The Reynolds number 


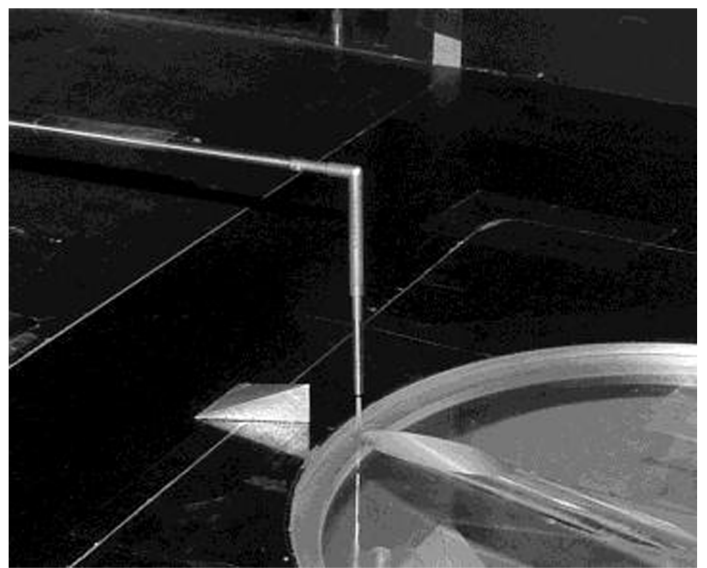

a)

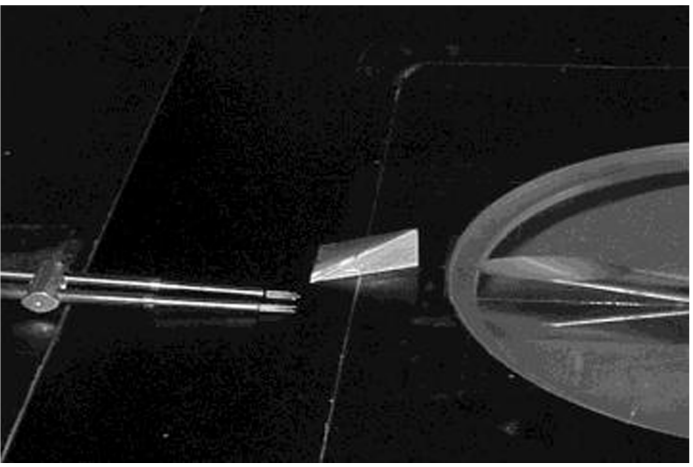

b)

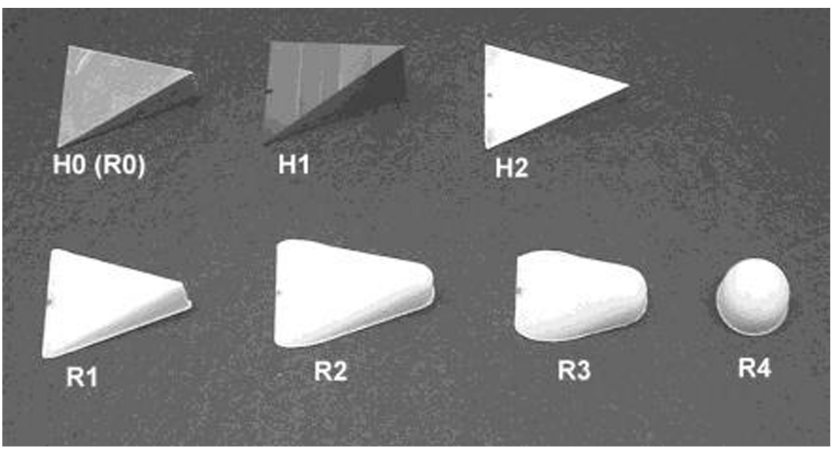

c)

Fig. 1 Experimental setup in wind tunnel: a) orifice and VG with single hot wire, b) two X-wire setup, and c) VG models.
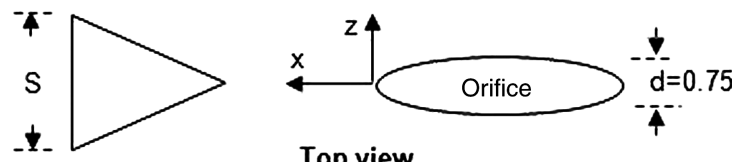

Top view

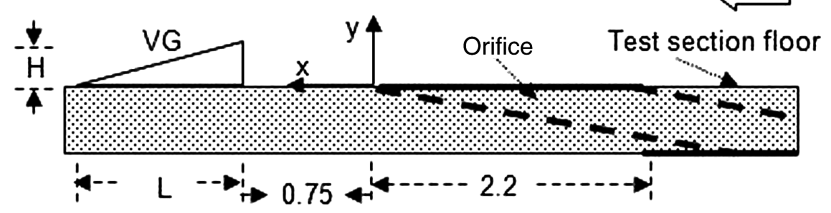

Side view

Fig. 2 Schematic of VG and orifice with coordinate system. Dimensions are in inches.
Table 1 Dimensions of VG models (Fig. 1c)a-

\begin{tabular}{cc}
\hline \hline Notation & Parameter \\
\hline VG & $H($ in $)$ \\
H0 & 0.561 \\
H1 & 1.125 \\
H2 & 0.281 \\
$(L=1.435$ in, $S=1.18$ in, $\rho=0.005$ in $)$ \\
VG & $\rho$ (in) \\
R0 & 0.005 \\
R1 & 0.032 \\
R2 & 0.141 \\
R3 & 0.250 \\
R4 & 0.340 \\
$\left(H=0.561\right.$ in, area blockage $\left.=0.33 \mathrm{in}^{2}\right)$ \\
\hline
\end{tabular}

${ }^{\mathrm{a}} \rho$ is the radius of curvature of the edges of the VG.

$R e$ based on freestream velocity and the orifice diameter is 11,400 . Both the $R e$ and $\delta / d$ are approximately the same as used in [1]. A single hot wire is used for preliminary checkouts, as well as overthe-orifice measurements (Fig. 1a). Two $X$ wires, one in the $u-v$ configuration and the other in the $u-w$ configuration (Fig. 1b), are used for detailed flowfield surveys downstream of the VG. Standard constant-temperature anemometry was used, and further details of the measurement procedure may be found in [6]. In the cited reference, uncertainty in the streamwise velocity and vorticity were estimated to be 2 and 20\%, respectively; except for regions immediately downstream of the orifice or the VG, the same holds true in the present experiment. The surveys are conducted on $y-z$ planes at various $x / d$ locations. Detailed surveys are done for the $\mathrm{H} 0$ model

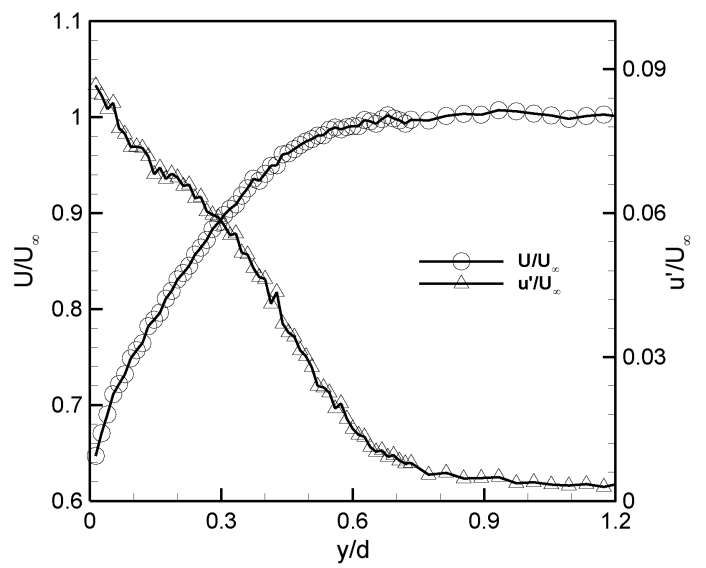

Fig. 3 Approach boundary-layer profiles measured at $x / d=-2.95$ and $z=0 . U_{\infty}=29 \mathrm{ft} / \mathrm{s}$.
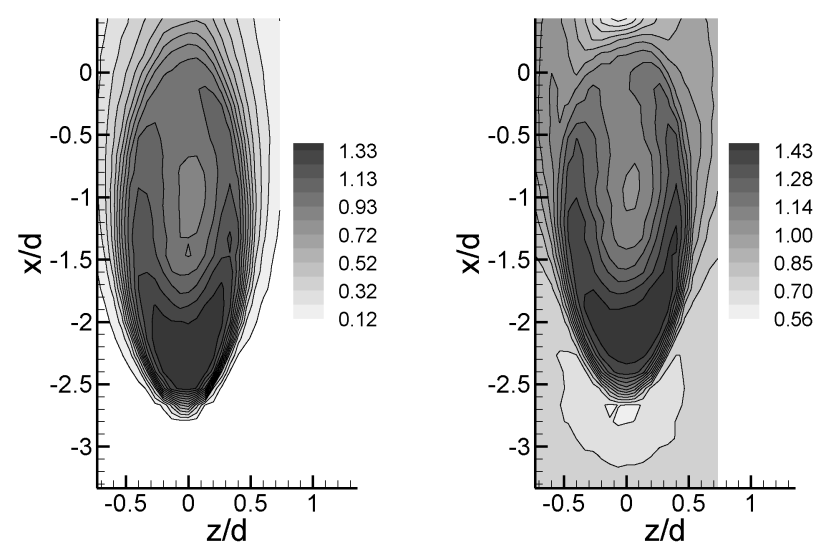

a)

b)

Fig. 4 Mean velocity distribution at orifice exit $(y / d=0.04)$; velocities are normalized by a reference velocity of $29 \mathrm{ft} / \mathrm{s}$ : a) $U_{\infty}=0$ and $U_{j}=41 \mathrm{ft} / \mathrm{s}$, and b) $U_{\infty}=29 \mathrm{ft} / \mathrm{s}$ and $U_{j}=41 \mathrm{ft} / \mathrm{s}$. 

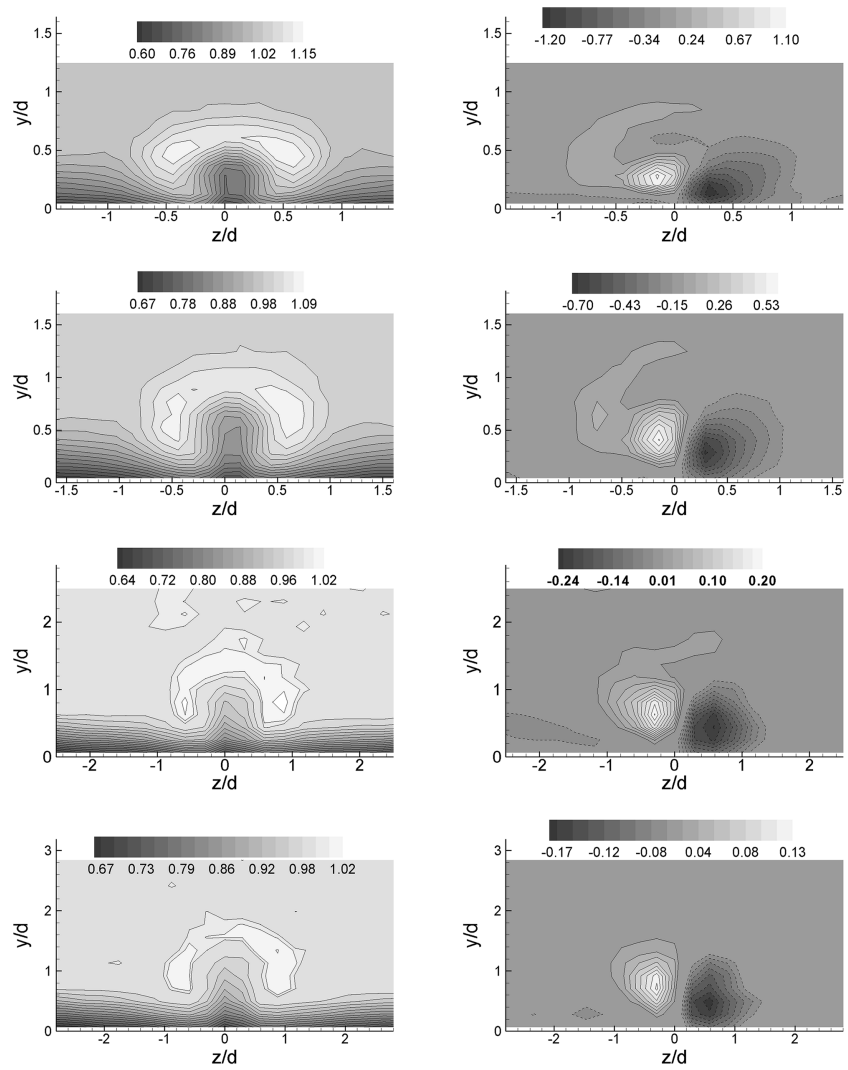

a)

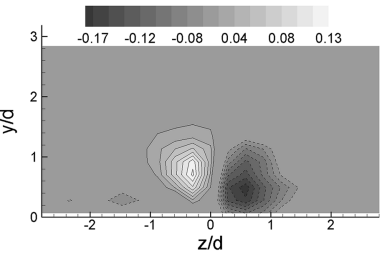

b)

Fig. 5 Flowfield properties for JICF-only case. In each figure, from top to bottom, the data are for surveys at $x / d=3.1,5,10$, and 15. a) Mean velocity $\left(U / U_{\infty}\right)$, and b) streamwise vorticity $\left(\omega_{x} d / U_{\infty}\right)$.
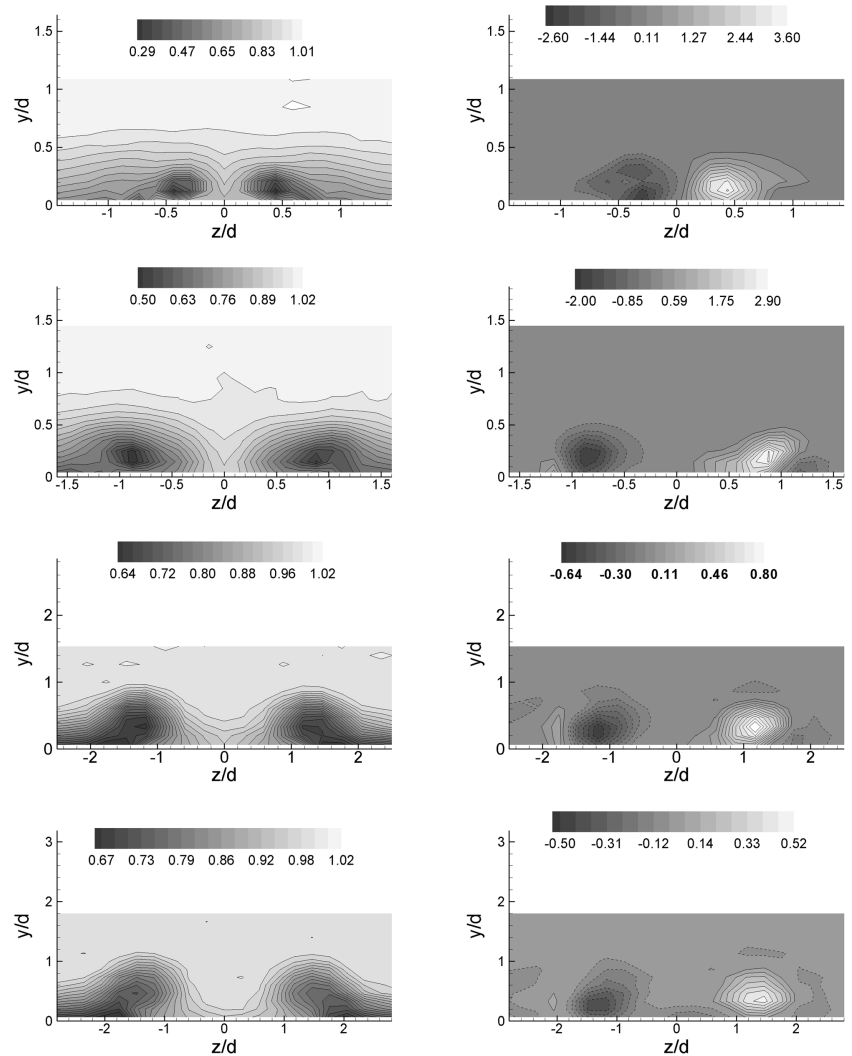

a)

Fig. 6 Flowfield properties for VG-only case. In each figure, from top to bottom, the data are for surveys at $x / d=3.1,5,10$, and 15. a) Mean velocity $\left(U / U_{\infty}\right)$, and b) streamwise vorticity $\left(\omega_{x} d / U_{\infty}\right)$. with its tip placed at $x / d=1$. The surveys are done at $x / d=3.1,5$, 10 , and 15, the first location being at the foot (i.e., at the left edge of the VG) (Fig. 2). With parametric variations, the surveys are conducted mainly at $x / d=10$ for diagnosing the relative effect. Data for most cases are taken for three conditions: 1) only JICF without the VG, 2) only VG without the JICF, and 3) a combination of VG and JICF.

Note that, while data for all three components of mean velocity and turbulent stresses have been acquired, for brevity, mostly data for streamwise mean velocity and vorticity are presented. Only examples of detailed contour maps are shown, while overall trends for these flow properties as well as turbulence intensity are summarized by line graphs. An interested reader may find more details in the conference version of this paper [7]. Note also that, unless stated otherwise, the data pertain to a nominal freestream velocity $U_{\infty}$ of $29 \mathrm{ft} / \mathrm{s}$ and a jet velocity $U_{j}$ of $41 \mathrm{ft} / \mathrm{s}$, yielding, for the present incompressible flow, a momentum flux ratio $J\left[=\left(U_{j} / U \infty\right)^{2}\right]$ of 2 . Furthermore, unless stated otherwise, the data pertain to the model H0 (or R0) with its tip located at $x / d=1$.

\section{Results}

The mean velocity distributions over the orifice, as measured by a single hot wire, are shown in Fig. 4. The plot in Fig. 4a represents data for only the jet flow with the tunnel flow turned off. The plot in Fig. $4 \mathrm{~b}$ shows corresponding data with the addition of the tunnel flow. Note that the high velocity is concentrated near the upstream end of the orifice. There is a noticeable minimum in the center of the orifice and generally lower velocities on the downstream end. With the tunnel flow turned on, the distributions basically remain the same. Regions just upstream and downstream of the orifice are marked by lower velocities, apparently because of the blockage created by the JICF. A slight asymmetry remained in the jet despite efforts to remedy it by tracking its source. As it will be seen, the effect of the VG dominates the flow, and the asymmetry in the jet may be considered inconsequential.
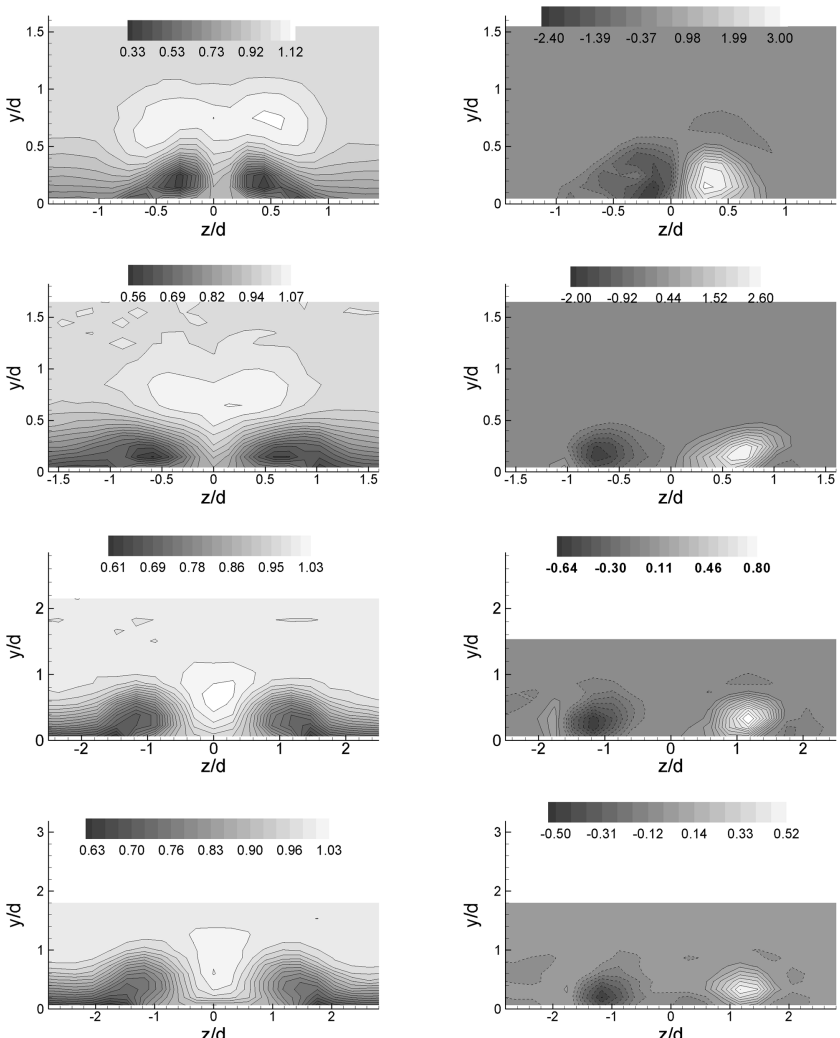

a)

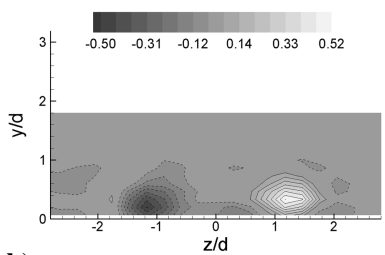

b)

Fig. 7 Flowfield properties for VG-plus-JICF case. In each figure, from top to bottom, the data are for surveys at $x / d=3.1,5,10$, and 15 . a) Mean velocity $\left(U / U_{\infty}\right)$, and b) streamwise vorticity $\left(\omega_{x} d / U_{\infty}\right)$. 


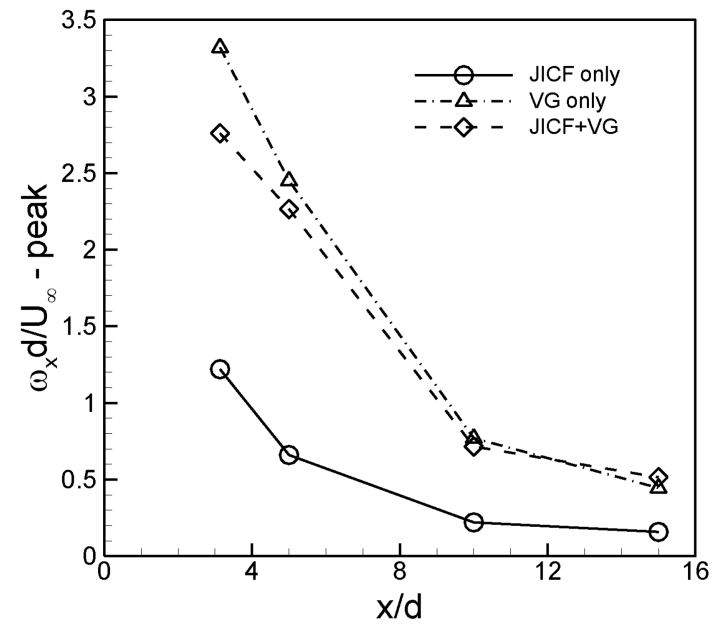

a)

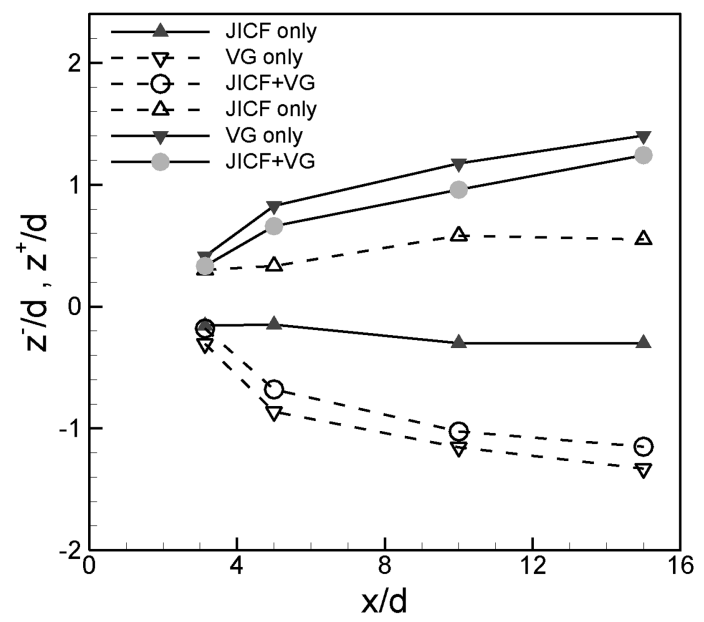

b)

Fig. 8 Variation of streamwise vorticity properties with distance for indicated conditions: a) peak amplitude, and b) trajectories of vortex cores.

Figure 5 documents the evolution of the mean velocity and streamwise vorticity for the JICF-only case. The surveys are for four axial locations indicated in the caption. As stated in Sec. II, these data are for model $\mathrm{H} 0$ with its tip at $x / d=1$ and for $J=2$. Note that the walls of the test section are $20 d$ away on either side; thus, wall effects may be considered negligible even at the last station. The mean velocity distributions exhibit the dome of high-speed jet fluid, which would be the coolant fluid in the film-cooling application. With the given orifice, inclined at $20^{\circ}$, the jet has already lifted up from the wall, even at the closest measurement location of $x / d=3.1$ (top, Fig. 5a). Corresponding streamwise vorticity data, shown in Fig. 5b, capture the evolution of the bound vortex pair. The sense of this vortex pair is such that there is an upwash in between the two vortices. The vorticity amplitudes drop off rapidly with increasing distance, but the vortex pair is unambiguously discernible even at the last measurement station.

Similar data for the VG-only case are shown in Fig. 6. A large effect is seen on the mean velocity distribution. Boundary-layer fluid is pushed apart while freestream fluid is brought in the middle to fill the space. This occurs due to the action of a strong vortex pair originating from the VG, as seen in Fig. 6b. The peak vorticity amplitudes in this case are about three times larger than those seen with the JICF in Fig. 5 . Note also the change in sense. Here, the sense is such that there is a downwash between the two vortices.

Corresponding data for the JICF-plus-VG case are shown in Fig. 7. It is evident from the mean velocity distributions that the jet fluid is pulled down toward the wall. This occurs due to the action of the

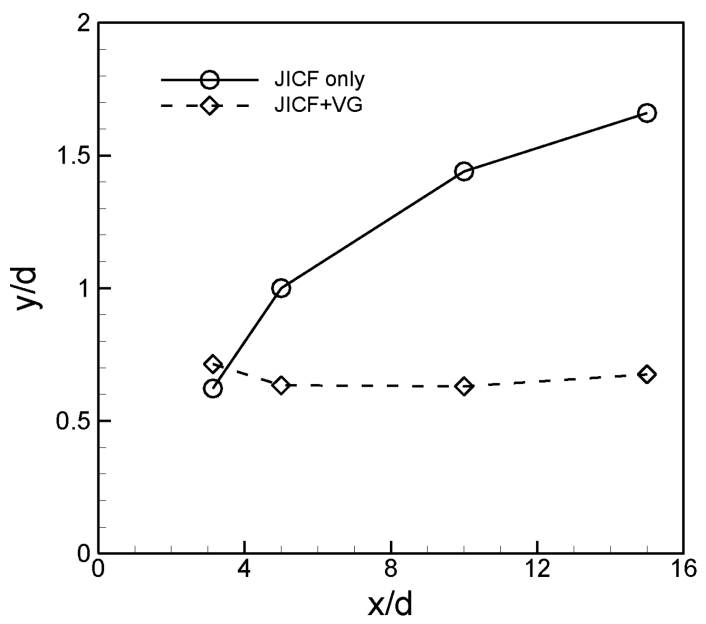

a)

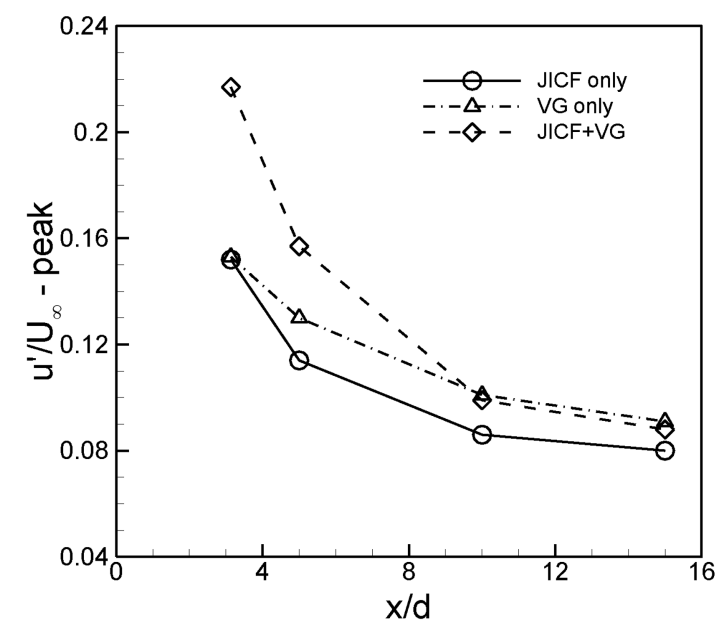

b)

Fig. 9 Variation of streamwise velocity and turbulence intensity with distance for indicated conditions: a) peak mean velocity on $z=0$ plane (jet penetration), and b) magnitude of peak turbulence intensity in the field.

streamwise vortex pair from the VG that dominates the flow (Fig. 7b). Thus, in the film-cooling application, the VG would be beneficial, since the coolant (jet) flow is brought closer to the wall. As stated in Sec. II, data on turbulent stresses were also acquired. The turbulence intensity distributions appear similar to the mean velocity distributions with peak intensities occurring in regions of a maximum $U$ gradient. Detailed data on turbulence are not shown for space conservation, and only overall trends based on line graphs are discussed in the following.

The overall trends from Figs. 5-7 are captured in the profiles and trajectories shown in Figs. 8 and $\overline{9}$. The variation of peak streamwise vorticity is shown in Fig. 8 a . The average of the absolute magnitudes in the cores of the two vortices in a pair is taken as the peak value. Initially, the peak value for the VG-plus-JICF case is somewhat smaller relative to the VG-only case, as expected since there is some vorticity cancellation in the combined case. Note again that the vortices with the VG are much stronger relative to those of the JICF. If the vortices from the VG were of the same magnitude as those of the JICF (but opposite sign), would the cancellation be maximum? Would this result in a better effectiveness of the VG? The issue is addressed later in the paper. The trajectories of the streamwise vortex cores are shown in Fig. 8b. There is more lateral spreading with the stronger vortices for the $\bar{V} \mathrm{G}$ cases relative to that for the JICF-only case.

The trajectories of the peak mean velocity on the $z=0$ plane are shown in Fig. 9a. These data show the jet penetration and provide a clearer idea about how much the JICF (coolant) core has been drawn 

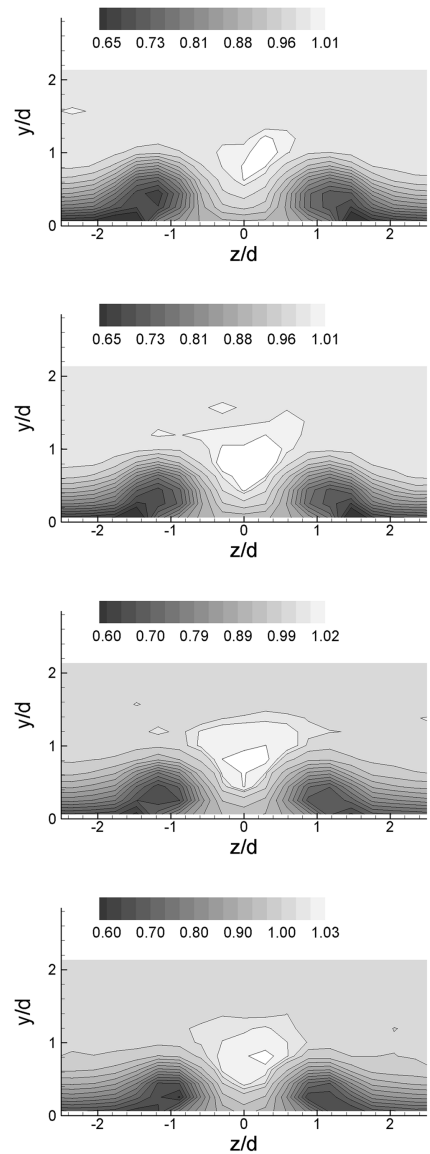

Fig. 10 Mean velocity $\left(U / U_{\infty}\right)$ distribution measured at $x / d=10$ for varying position of VG. From top to bottom, VG tip positions $\left(x_{T} / d\right)$ are $0,0.5,2$, and 3 .

toward the wall. It is needless to emphasize that the influence of the VG may appear too good from this simplistic graph. The flowfield in its entirety (Figs. 5-7) must be considered to assess the overall effect. Figure $9 \mathrm{~b}$ shows the variation of the peak turbulence intensity with downstream distance. At each $x$ station, the maximum level in the field is taken as the peak value. When the JICF is combined with the
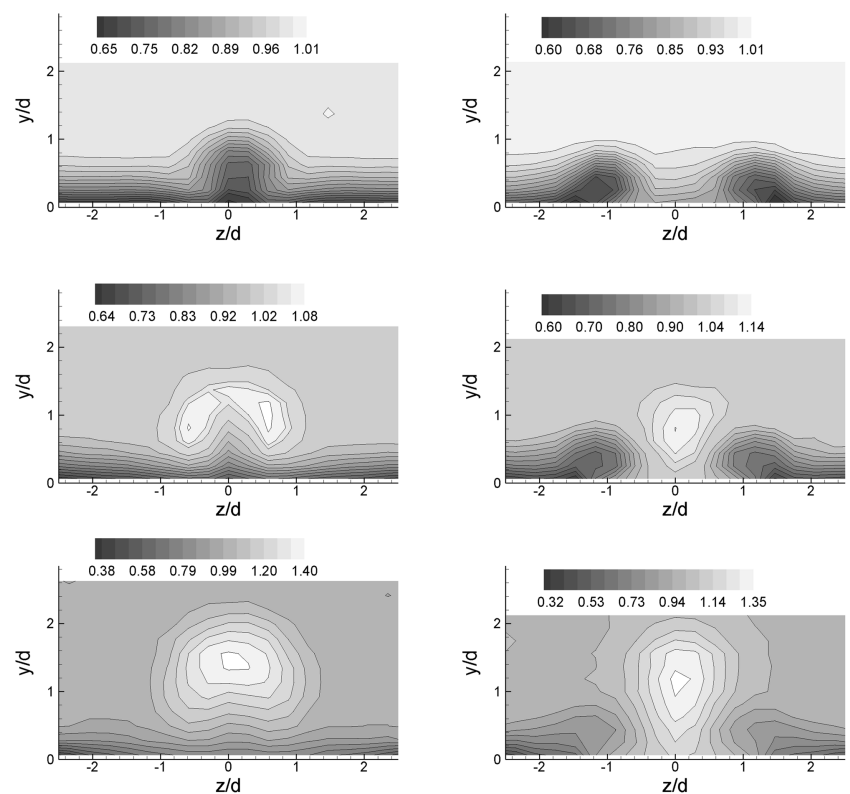

a)

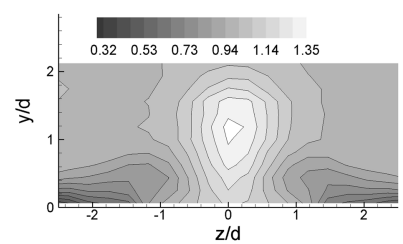

b)

Fig. 11 Mean velocity $\left(U / U_{\infty}\right)$ distribution measured at $x / d=10$ for different momentum flux ratio $(J)$. In each figure, the data from top to bottom are for $J=1,3$, and 6.7: a) JICF only, and b) VG plus JICF.
VG, the initial peak level is much larger. The level becomes comparable to that for the VG-only case farther downstream. While the VG clearly pulls the JICF toward the wall, the high initial turbulence may have an adverse effect from the film-cooling perspective. The JICF (i.e., coolant flow) core might dissipate faster due to turbulent diffusion. In fact, an effect like this can be seen in Fig. 7a. While the core is brought closer to the wall, the spatial extent

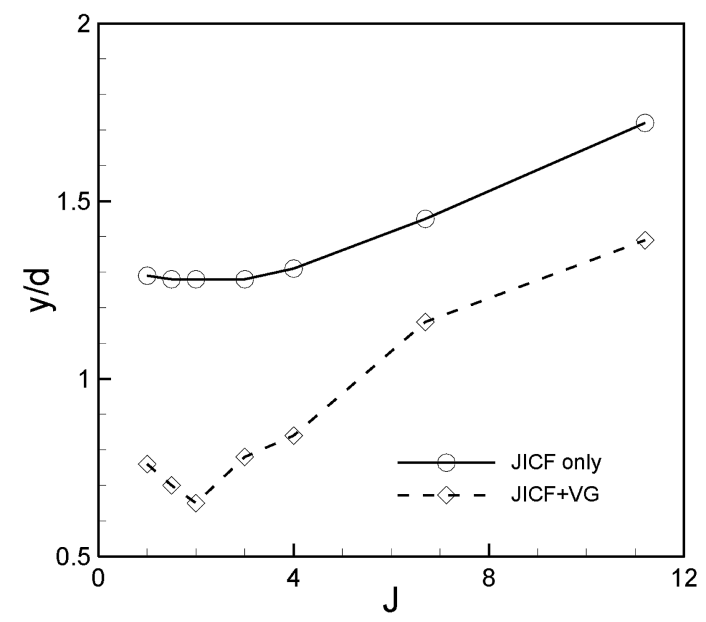

a)

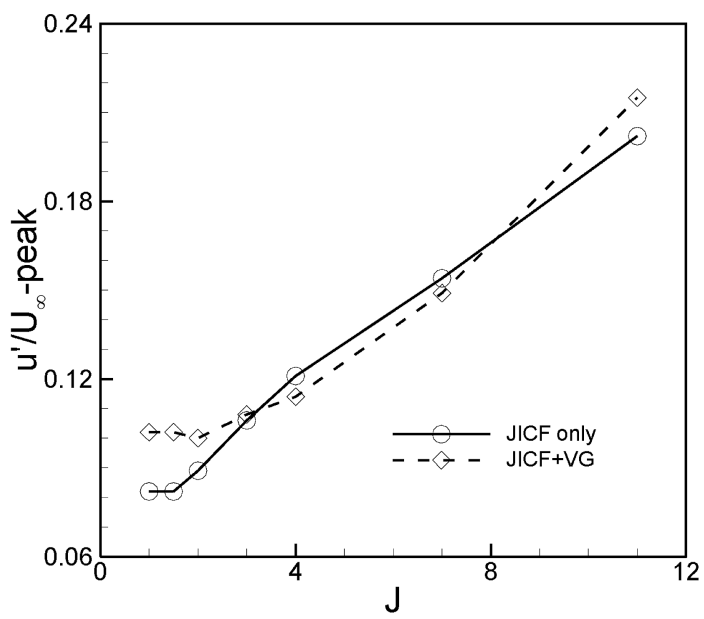

b)

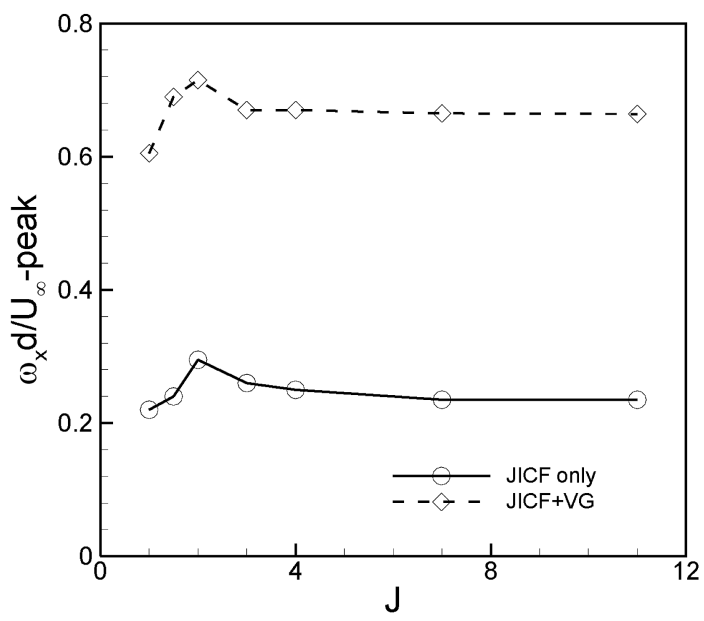

c)

Fig. 12 Variation of flowfield properties with $J$ measured at $x / d=10$ : a) location of peak mean velocity at $z=0$ (jet penetration), b) peak turbulence intensity, and c) peak streamwise vorticity (solid curves: JICF only case; dashed curves: JICF-plus-VG case). 
of the core (bright region, say, at $x / d=15$; at bottom of Fig. 7a) is smaller than the corresponding region for the JICF-only case (at bottom of Fig. 5a). Thus, the application of the VG would require a balance. On one hand, a strong enough streamwise vortex pair must be generated to effectively pull the jet toward the wall. On the other hand, the action of the VG must not be too strong to generate excessive turbulence that will dissipate the jet fluid quickly.

The effect of placing the VG (model H0) at a different distance from the orifice is shown in Fig. 10. These surveys are for the fixed location of $x / d=10$. Only mean velocity data are shown for brevity. The graph at the top is for the VG with its apex placed right at the end of the orifice; the bottom graph is for the farthest location of the VG. It is apparent that varying the VG location does not affect the velocity field significantly. Corresponding vorticity data show a similar distribution, as seen in Fig. 7. As the VG is moved downstream, $\omega_{x}$ cores are drawn somewhat closer together (lateral distance decreases from $2.2 d$ at zero separation to $1.8 d$ at the farthest separation), and the magnitude of $\omega_{x} d / U_{\infty}$ peak increases somewhat (from 0.7 at zero separation to 1.1 at the farthest separation). Perhaps, these trends are expected, since the measurement station becomes closer as the VG is drawn downstream. In any case, the changes are small relative to those produced by other parametric variations, and one has to infer that the flowfield is rather insensitive to the exact location of the VG.

The effect of varying the momentum flux ratio $J$ over the range of 1-11 was explored. Unfortunately, the control for the jet flow was coarse, and additional data on the lower end of the range, more typical of film-cooling application, could not be taken. The parameter $J$ was varied by varying $U_{\infty}$ while keeping $U_{j}$ a constant. The mean velocity distributions are shown in Fig. 11 a for the JICFonly case for three values of $J$, as examples. At all $J$, there is jet liftoff at the measurement station.
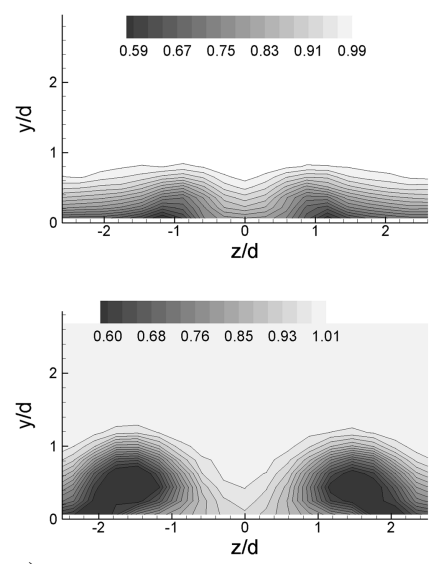

a)
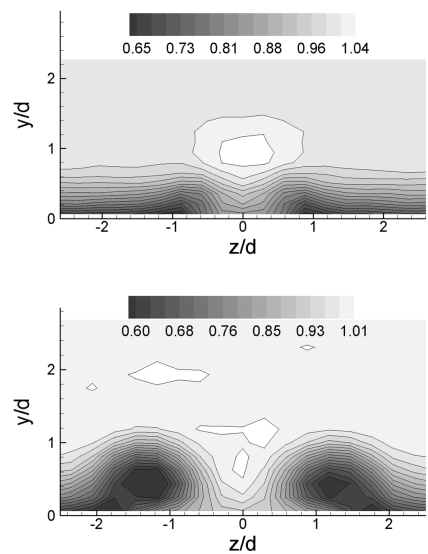

b)

Fig. 13 Mean velocity $\left(U / U_{\infty}\right)$ distribution measured at $x / d=10$ for different VG heights $H$. In each figure, the data from top to bottom are for $H / d=0.375$ and 1.50: a) VG only, and b) VG plus JICF.
Corresponding data for the VG-plus-JICF case are shown in Fig. $11 \mathrm{~b}$. It is apparent that, even at the highest $J$, the jet core is brought significantly closer to the wall by the action of the VG. Overall trends from the mean velocity, turbulence, and vorticity distributions, as a function of $J$, are summarized in Fig. 12. The location of the peak of $U$ on the $z=0$ plane (jet penetration) is shown in Fig. 12a. It can be seen that the high-speed jet fluid is brought closer to the wall even at the highest value of $J$ covered in the experiment. Corresponding peak turbulence intensity profiles are shown in Fig. 12b. At small values of $J$ (pertinent to film-cooling application), the intensity increases with the addition of the VG, as noted earlier with Fig. 9b. However, at higher $J$, the relative change in the intensity is not as much. The peak streamwise vorticity amplitude, shown in Fig. 12c, is much larger when the VG is added, and this trend remains valid at all $J$. The amplitude exhibits a peak around $J=2$ and then remains invariant at higher values of $J$ in either case of Fig. 12c.

All data presented so far pertained to the model H0. Data taken for two other VG heights (cases H1 and H2, Table 1), are shown in Fig. 13. For the VG-only case in Fig. 13a, it can be seen that the height has a profound effect. The velocity defects and the indentation in the middle are larger with increasing $H$. Corresponding data for the VG-plus-JICF case (Fig. 13b) exhibit a similar trend. Here, we note that the VG with the smaller height has been ineffective, and there is a liftoff of the jet fluid at the measurement station. On the other hand, for the VG with the larger height, the jet core has practically diffused due to effect from large turbulence. Thus, for the range covered, the intermediate height $(H=0.75 d$; Fig. $7 \mathrm{a})$ appears to be most effective in pulling the jet toward the wall while not dissipating it adversely.

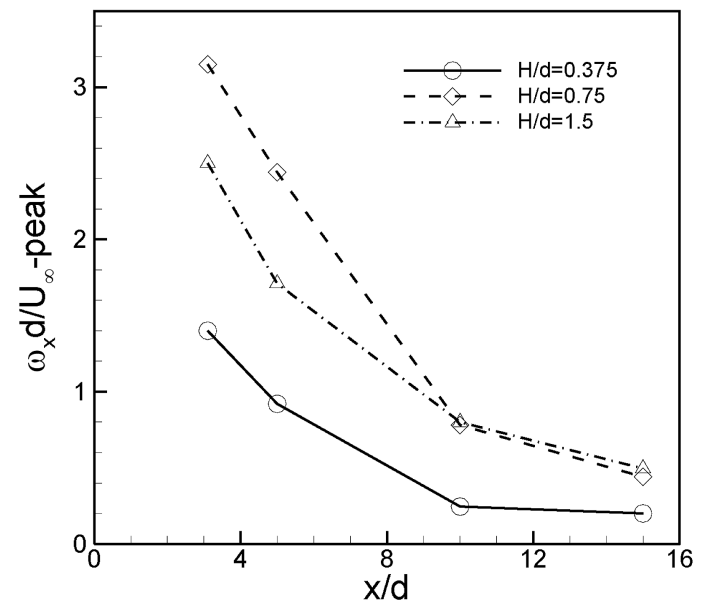

a)

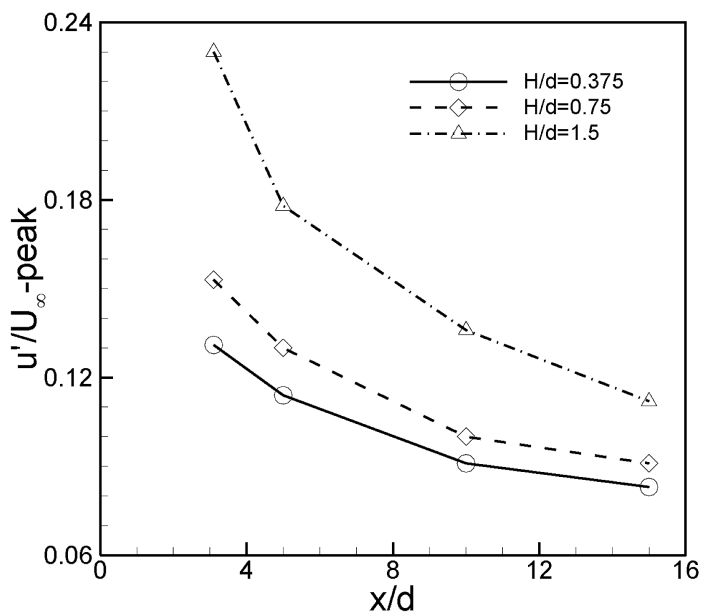

b)

Fig. 14 Variation of flowfield properties with $x / d$ for different VG heights $H$ : a) peak streamwise vorticity, and b) peak turbulence intensity. 
From an applications point of view, a smaller size of the VG would be desirable to minimize losses. The total pressure loss due to the VG could not be measured conveniently in the experiment. This issue is to be addressed in a complimentary computational study expected to be carried out in the near future.

The overall effect of increasing VG height on peak vorticity and turbulence intensity is captured in Fig. 14. It is interesting to note that the intermediate height case produced the largest peak vorticity at the upstream locations. An inspection of the vorticity maps for the larger height case, at locations close to the VG, indicate that the vorticity is distributed over a larger region. With increasing $x / d$, the vorticity for this case amalgamates to form a tighter core. Thus, peak amplitudes measured farther downstream become comparable with that for the intermediate case. With reference to earlier discussion (Fig. 8a), we note that the smallest height case has produced streamwise vortices that are comparable in magnitude (but opposite in sense) relative to the JICF-only case; compare the solid curves between Figs. 8a and 14a. Yet, this VG proved to be ineffective. Thus, vorticity magnitudes significantly stronger than the JICF-only case must be produced in order for the VG to be effective.

Finally, the effect of rounding off the edges of the VG is shown in Figs. 15 and 16. Mean velocity distributions for the VG-only case are shown in Fig. 15a. The three sets of data are for the R1, R2, and R4 models (Table 1 ). It is noteworthy that even with the limiting hemispherical case ( $\left.\mathrm{R} 4 ; \rho / \rho_{4}=1\right)$, a similar velocity distribution, with an inward indentation in the middle, is observed. Vorticity data for this case clearly exhibit a streamwise vortex pair of the same (downwash) sense, albeit with smaller magnitude [7]. Corresponding data for the VG-plus-JICF case are shown in Fig. $15 \mathrm{~b}$. It is apparent that, with the jet, the flow pattern has changed for the R4 model. The jet flow has overwhelmed the effect of the VG, and the basic feature of the JICF has taken over (compare with JICF-only case in Fig. 5). A vortex pair with a small magnitude but an upwash sense has prevailed in the latter case.

The streamwise vorticity magnitudes for the R-series models are analyzed in Fig. 16. Peak $\omega_{x}$ values are shown as a function of the radius of curvature used to round off the edges. (As stated earlier, the average of two absolute peaks from a pair is taken for these data, with the downwash sense being assigned a positive sign and the upwash
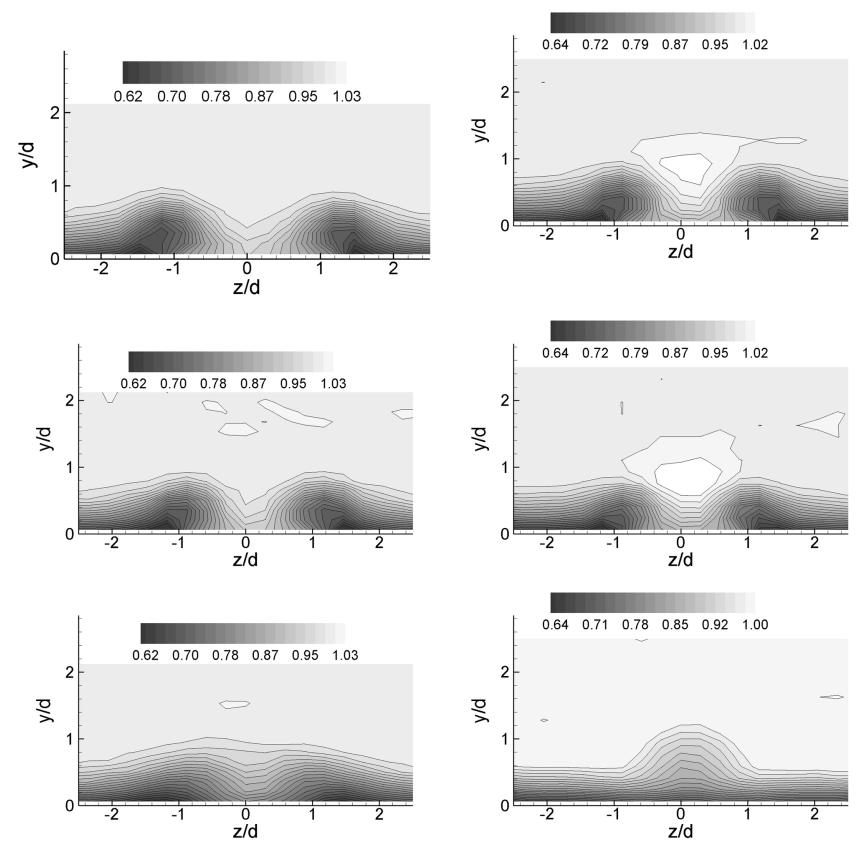

a)

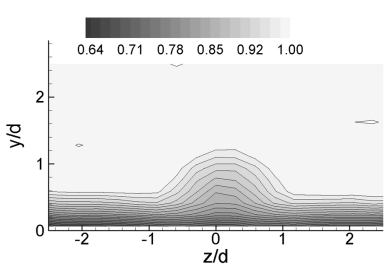

b)

Fig. 15 Mean velocity $\left(U / U_{\infty}\right)$ distribution measured at $x / d=10$ for varying roundedness of the edges of $\mathrm{VG}\left(\rho / \rho_{4} ; \rho_{4}\right.$ is radius of curvature for the fully rounded model R4). In each figure, from top to bottom, the data are for $\rho / \rho_{4}=0.015,0.415$, and 1.00: a) VG only, and b) VG plus JICF.

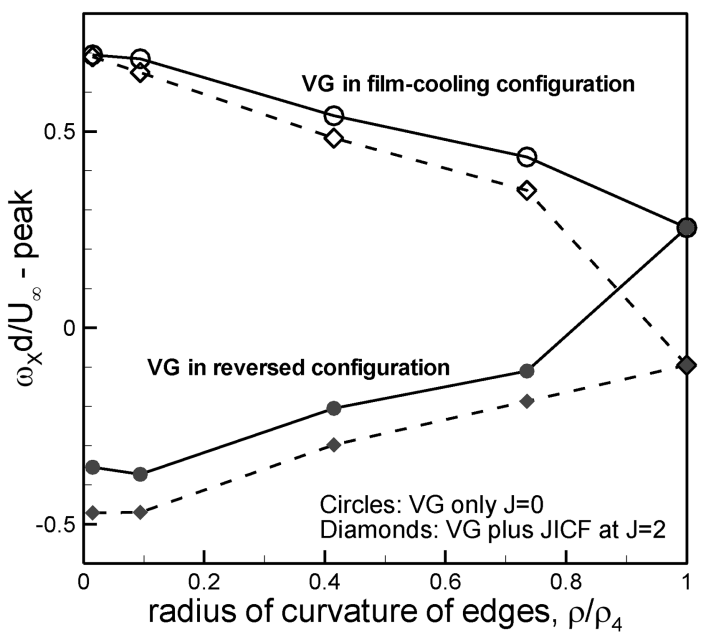

Fig. 16 Effect of varying roundedness of the edges of VG on peak streamwise vorticity at $x / d=10$. Upper two curves are for film-cooling configuration of VG as used for all previous data. Lower two curves are for the case with VG orientation reversed by $180^{\circ}$ (solid curves: VG-only case; dashed curves: VG-plus-JICF case).

sense being assign a negative sign). Data for cases with a reversed placement of the VG are also included in this figure. The VG-only case is represented by the circular symbols with the solid lines. Open points are for the film-cooling configuration, while the solid points are for the reversed configuration. As expected, the reversed configuration produces vortex pairs of opposite sense (negative ordinate). It can be seen that the absolute magnitudes of peak $\omega_{x}$ are significantly larger in the film-cooling configuration. It is as if the flow favors the downwash sense, producing a pair of that sense even in the limiting hemispherical case. This suggests that even the hemispherical model should be successful in pulling the jet down toward the wall to some extent. This corroborates the observation in [8] that a deposition in front of the cooling orifice produces a beneficial effect. Streamwise vorticity data have rarely been reported in the literature for comparable flows. Reference [9] reports detailed velocity measurements behind a bump placed in a turbulent boundary layer, and some similarity in the velocity distributions could be observed between their data and the present data.

The dashed curves with the diamond symbols in Fig. 16 represent the peak vorticity data for the VG-plus-JICF case. Obviously, with the addition of the JICF, there is vorticity cancellation in the filmcooling configuration but augmentation with the reversed configuration, resulting in a downward shift of the entire loop. For the limiting hemispherical model (data on far right), the magnitude has dropped more than that for the other radii. From the overall trends in this figure, it is amply clear that rounding off the VG edges has a significant effect. With increasing roundedness, there is decreasing amplitude of $\omega_{x}$ and, therefore, less effectiveness of the VG. A small amount of rounding (e.g., second data point from left with model R1), however, may be quite tolerable with minimal impact on the effectiveness of the VG.

\section{Conclusions}

Experimental results on the interaction of a VG with an inclined JICF are presented in this paper. The VG produces a streamwise vortex pair that has a sense opposite to that of the bound vortex pair in the JICF. Thus, placing the VG downstream of the JICF has a vorticity cancellation effect with a resultant retardation in the tendency for the jet to lift off from the wall. A VG model with dimensions comparable to the orifice diameter and the approach boundary-layer thickness is found to produce streamwise vorticity approximately three times larger in magnitude than that found with the JICF alone. This configuration appears to be most effective in keeping the flow attached to the wall. By measuring the flowfield at 10 diameters from the orifice, the following observations are made. 
When the height of the VG is halved, there is a liftoff of the jet. On the other hand, when the height is doubled, the jet core is found to be dissipated due to larger turbulence intensities. Varying the location of the VG over three diameters from the orifice has little impact on the flowfield at the measurement station. It is found that even at the highest value of $J(=11)$ covered in the experiment, the VG is quite effective in pulling the jet toward the wall. Finally, it is found that the edges of the VG need to be sharp in order to be effective. With the increasing radius of curvature of the edges, the vortices weaken, and the VG becomes less effective. However, a small radius of curvature may be quite tolerable.

\section{Acknowledgment}

This work is supported by the Subsonic Fixed Wing Project of the NASA Fundamental Aeronautics Program.

\section{References}

[1] Rigby, D. L., and Heidmann, J. D., "Improved Film Cooling Effectiveness by Placing a Vortex Generator Downstream of Each Hole," ASME Gas Turbine Conference, American Society of Mechanical Engineers Paper 2008-GT-51361, Fairfield, NJ, June 2008.

[2] Hirt, S. M., and Anderson, B. H., "Experimental Investigation of the Application Of Microramp Flow Control to an Oblique Shock Interaction," 47th Aerospace Sciences Meeting, AIAA Paper 20090919, Jan. 2009.
[3] Ford, C. W. P., and Babinsky, H., "Micro-Ramp Control of Oblique Shock Wave/Boundary Layer Interactions," 37th Fluid Dynamics Conference, AIAA Paper 2007-4115, June 2007.

[4] Zaman, K. B. M. Q., "Reduction of Jet Penetration in a Cross-Flow by Using Tabs," 34th Joint Propulsion Conference, AIAA Paper 19980327, July 1998 .

[5] Heidmann, J. D., and Ekkad, S., "A Novel Antivortex Turbine FilmCooling Hole Concept," Journal of Turbomachinery, Vol. 130, No. 3, July 2008, Paper 031020 . doi:10.1115/1.2777194

[6] Milanovic, I. M., and Zaman, K. B. M. Q., "Fluid Dynamics of Highly Pitched and Yawed Jets in Crossflow," AIAA Journal, Vol. 42, No. 5, 2004, pp. 874-882. doi: $10.2514 / 1.2924$

[7] Zaman, K. B. M. Q., Rigby, D. L., and Heidmann, J. D., "Experimental Study of an Inclined Jet-In-Cross-Flow Interacting with a Vortex Generator," 48th AIAA Aerospace Sciences Meeting, AIAA Paper 2010-0088, Jan. 2010.

[8] Sundaram, N., and Thole, K. A., "Effects of Surface Deposition, Hole Blockage, and TBC Spallation on Vane Endwall Film-Cooling," ASME Turbo Expo, American Society of Mechanical Engineers Paper GT90379, Fairfield, NJ, May 2006.

[9] Byun, G., and Simpson, R. L., "Structure of Three-Dimensional Separated Flow on an Axisymmetric Bump," 43rd Aerospace Sciences Meeting, AIAA Paper 2005-0113, Jan. 2005. 